\title{
A study protocol for expanding the screening interval of endoscopic screening for gastric cancer based on individual risks: prospective cohort study of gastric cancer screening
}

\author{
Chisato Hamashima $^{1}$, Kenichi Yoshimura ${ }^{2}$, Akira Fukao ${ }^{3} \wedge$ \\ ${ }^{1}$ Health Policy Section, Department of Nursing, Faculty of Medical Technology, Teikyo University, Tokyo, Japan; ${ }^{2}$ Future Medical Center, \\ Hiroshima University Hospital, Hiroshima 734-8551, Japan; ${ }^{3}$ Miyagi Cancer Association, Sendai, Japan \\ Correspondence to: Chisato Hamashima, MD, DrMedSc. Health Policy Section, Department of Nursing, Faculty of Medical Technology, Teikyo \\ University, 2-11-1 Kaga, Itabashi-ku, Tokyo 173-1211, Japan. Email: chamashi@med.teikyo-u.ac.jp.
}

Background: The Japanese government has recommended a 2-year endoscopic screening interval for gastric cancer. However, insufficient resources have constrained participation in endoscopic screening for gastric cancer. One way to avoid endoscopic screening harms and provide equal access is to define the appropriate screening interval.

Methods: To expand screening interval from more than 2 years for low-risk group, a single-arm cohort of endoscopic screening started. At the baseline screening, the participants underwent endoscopic screening for gastric cancer, Helicobacter pylori (H. pylori) antibody test, and serum pepsinogen test (first year), and followed after 2 and 4 years (within the first 5 years). We also assessed $H$. pylori infection and atrophy status on images of upper gastrointestinal endoscopy at the baseline. A new screening model will be developed by dividing the participants into high-risk and low-risk groups based on demographics, history of $H$. pylori eradication, serological testing, and endoscopic diagnosis. The cumulative gastric cancer incidence after negative results at baseline are compared between the low-risk group on the 3 rd screening round after 4 years from baseline and the total screening group on the 2 nd screening round after 2 years. If the cumulative gastric cancer incidence in the low-risk group on the 3 rd screening round is lower than that in the total screening group on the 2 nd screening round, the screening interval can be expanded to 4 years in the low-risk group.

Discussion: To reduce mortality from gastric cancer, a high participation rate of the target population is required. The screening interval of endoscopic screening can be changed if the individual risks for $H$. pylori infection are clarified. Our goal in this study is to obtain relevant data that can be used to improve the efficient use of endoscopic screening for gastric cancer by referring to individual risks in Japan.

Trial registration: UMIN000025839 (University Hospital Medical Information Network, Japan)

Keywords: Gastric cancer; cancer screening; endoscopic screening; screening interval; atrophic gastritis; Helicobacter pylori

Submitted Aug 18, 2020. Accepted for publication Nov 08, 2020.

doi: 10.21037/atm-20-5949

View this article at: http://dx.doi.org/10.21037/atm-20-5949

^ ORCID: Chisato Hamashima 0000-0003-2585-8479; Kenichi Yoshimura 0000-0001-7019-2480; Akira Fukao 0000-0003-4575-0369. 


\section{Introduction}

Although gastric cancer mortality in Japan has decreased over the last 3 decades, its burden remains. Gastric cancer is the second and the fourth leading cause of cancer death in men and women, respectively (1). It accounts for about $10 \%$ of all cancer deaths in Japan. Upper gastrointestinal series (UGI) for gastric cancer screening was originally developed in Japan and it subsequently became a standard screening method (2). UGI for gastric cancer screening has been conducted as a national program across Japan since 1983 under the law for cancer screening (3). However, the participation rates in gastric cancer screening in Japan has decreased over the last 2 decades, and it has remained at approximately $10 \%$ (4). On the other hand, the upper gastrointestinal endoscopic technique has become more commonly used among general physicians nationwide, with the number of endoscopic examinations increasing among medical offices (5). Thus, the introduction of endoscopic screening for gastric cancer has been expected. In 2016, the Japanese government introduced endoscopic screening as a national program based on the gastric cancer screening guidelines developed by the National Cancer Center of Japan (3).

Despite the rapid introduction of endoscopic screening in most urban cities, there has been some hesitation in regional municipalities because of the insufficient human resources. In these areas, endoscopic screening is mainly performed by general physicians. There are no dedicated rooms for endoscopic screening in regional areas, and this situation is aggravated by the insufficient number of physicians who can provide medical services (6). Therefore, to spread the use of endoscopic screening in Japan, the expansion of endoscopic capacity has become an urgent issue.

Although the screening interval of endoscopic screening was equally defined every 2 years for the target population, a different screening interval might be defined and considered depending on its relation to the individual's background and gastric cancer risk. Gastric cancer incidence differs according to individual risks and is mainly defined by $H$. pylori infection and atrophic gastritis $(7,8)$. If individuals with a low risk of gastric cancer could be identified and adopted to the programs, their screening interval can be expanded. This information would be useful in both avoiding the harms of screening and providing equal access to endoscopic screening nationwide in Japan. The purpose of this cohort study is to clarify the appropriate individual risks for endoscopic screening and then expand the screening interval for gastric cancer in the low-risk group.

\section{Methods}

\section{Study setting}

The proposed cohort involves multi-municipalities which have endoscopic screening program for gastric cancer. For implementation of endoscopic screening as national program, a quality assurance manual was developed (9) and it has been used in gastric cancer screening programs in regional areas. The ideal study areas that regularly follow the above-mentioned manual will be selected. Until 2019, the following municipalities in Japan that meet our requirements were recruited as study areas: Niigata, Kanazawa, Maebashi, Tottori, Yonago, Yuri-Honjyo, and Morioka. Also, Sendai, Setagaya-Tokyo, Yazu, and Fukuoka will be included in this study in 2020 .

\section{Study population}

The main target of gastric cancer screening is individuals aged from 50 to 60 years. Their incidence rate of those aged from 50-69 years is estimated to be $121.8(/ 100,000)(1)$. The subjects aged 50-69 years are recruited from the study areas. The eligibility criteria for participants in this study were as follows: (I) subjects aged 50-69 years at the year of recruitment, (II) inhabitants of the study area, (III) subjects who can participate in endoscopic screening for gastric cancer in local programs, and (IV) subjects who provide written informed consent to participate in this study. The exclusion criteria were as follows: (I) individuals who have cancer, (II) individuals with severe health conditions and unable to participate in endoscopic screening for gastric cancer, and (III) individuals who underwent gastric resection.

\section{Study design}

We performed a single-arm cohort study to assess the most appropriate endoscopic screening interval for gastric cancer in the low-risk group. The trial was registered at University Hospital Medical Information Network, Japan (UMIN000025839). The study was approved by the Ethics Committee of the Miyagi Cancer Association (No.1612, Date of approval $21^{\text {st }}$ March 2017). Each participant provided written informed consent before inclusion in the 


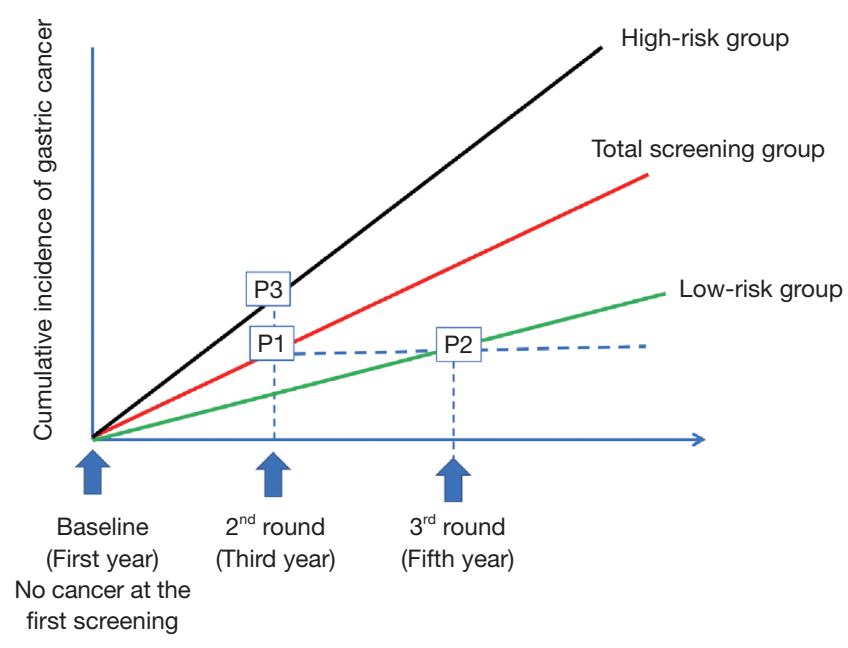

Figure 1 Basic concept of screening interval expansion. A new model that divides the participations into high-risk and lowrisk groups will be developed by referring to the participants' demographic information with reference to their history of $H$. pylori eradication, serological testing, and endoscopic diagnosis. If the cumulative incidence of gastric cancer in the low-risk group on the $3^{\text {rd }}$ assessment round (P2) becomes lower than the cumulative incidence of gastric cancer in the total screening group on the $2^{\text {nd }}$ assessment round (P1), the screening interval can be expanded from 2 to 4 years in the low-risk group.

study.

If individuals with a low risk of gastric cancer can be divided from individuals with a high risk of gastric cancer, the screening interval can be expanded. At this stage, the discrimination of individual risks for gastric cancer has not yet been strategically used in a gastric cancer screening program. Importantly, a new screening model can be developed regarding demographic information covering the history of $H$. pylori eradication, serological testing, and endoscopic diagnosis. The new screening model is expected to become more clearly delineated than serological testing alone. Participants without gastric cancer will continue to have endoscopic screening every 2 years. If the cumulative incidence in the low-risk group on the 3 rd screening round (P2) becomes lower than that in the total screening group on the 2nd screening round (P1), the screening interval can be expanded from 2 to 4 years in the low-risk group (Figure 1).

Individuals who agreed to participate in this study and provided written informed consent were confirmed as study subjects. They are recommended to undergo consecutive screenings for gastric cancer every 2 years within 5 years
(Figure 2). At the time of endoscopic screening, the participants were also asked to answer a questionnaire that covered the following: family history of gastric cancer, smoking habits, frequency of regular access to general physicians, history of UGI or endoscopy, diagnosis of gastric cancer, the history of $H$. pylori eradication, and EQ5D (10). After undergoing screening 3 times, a questionnaire survey is conducted at 7 years and 10 years after recruitment.

During the 10-year follow-up (Figure 2), gastric cancer incidence and mortality are confirmed using the official database. Population registers are also checked to confirm the inhabitants in the study area and their outward movement. For the primary outcome, the cumulative incidence of the low-risk group on the 3 rd screening round (P2) will be calculated and compared with total screening group on the 2 nd screening round (P1) (Figure 1). The secondary outcomes are the cumulative incidences of gastric cancer in the low-risk group and the high-risk group at 3,5 and 10 years.

\section{Data management and monitoring}

All study data is collected and maintained in regional management office which are mainly located in regional medical associations. The data includes informed consent forms, screening results, questionnaire survey and followup results. The regional management office revises them annually and submits the anonymous data to the central database. The principal investigators at the participating municipalities are responsible for collecting the medical research records from the screening database and population registries in their municipalities as well as reviewing and storing them. The central database maintains all data submitted from study areas. To maintain the quality of the database, regular checks are performed to identify missing or contradictory in their data.

\section{Sample size calculation}

Individuals who participate in endoscopic screening are divided into a high-risk group and a low-risk group for gastric cancer. Grading of atrophic gastritis was by the classification of Kimura and Takemoto (11). Borderline atrophic gastritis is defined as extending from the antrum toward the lesser curvature of the body and to the greater curvature. If the borderline atrophic gastritis does not reach the cardia, the atrophy grade is classified as a close type (C). 


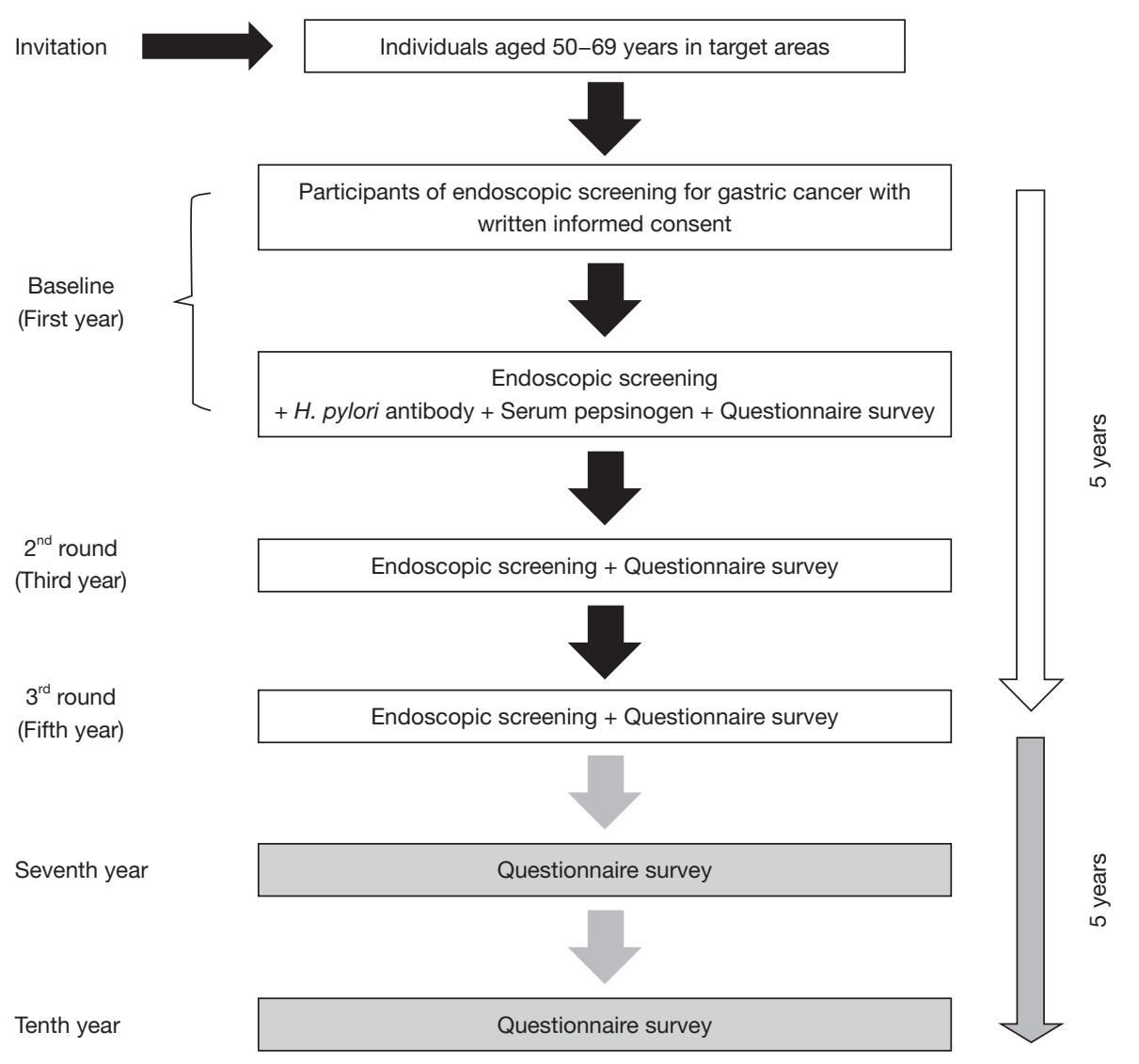

Figure 2 Screening flowchart schedule. The participants undergo endoscopic screening, $H$. pylori antibody test, and serum pepsinogen test at the first year, and then 2 endoscopic screenings in the third and fifth years within the first 5 years. After the 3 consecutive endoscopic screenings, follow-up will be continued in other years using a questionnaire survey for 5 years. After undergoing screening 3 times, a questionnaire survey is conducted at 7 and 10 years after recruitment.

If the borderline atrophic gastritis extends over the cardia, the atrophy is classified as an open type (O). Each group is further classified into 3 groups based on the atrophic areas: C-I, C-II, and C-III; O-I, O-II, and O-III. The distributions of atrophic gastritis in subjects undergoing endoscopic screening for gastric cancer have been reported in Kanazawa and Niigata, Japan. These places have conducted endoscopic screening for over 10 years $(12,13)$. Based on these previous studies, 2 cut-off values were assumed at $\mathrm{C}-\mathrm{II}$ and $\mathrm{O}-\mathrm{I}$ (Table 1). The distribution and cumulative incidence were defined for low and high groups in each scenario, respectively. The sample size was calculated to achieve $5 \%$ on both sides with an $80 \%$ power. In the first scenario, 19,844 subjects were needed. In the second scenario, 10,372 subjects were needed. If the followup rate is assumed as $80 \%, 12,965$ subjects are required for the second scenario.

\section{Statistical analysis plan}

We compared baseline characteristics of the high-risk and low-risk group by the Chi-square test, including sex, age, $H$. pylori infection, history of $H$. pylori eradication, history of gastric cancer screening and other demographic information which is obtained by a questionnaire survey. We examined the cumulative incidence of gastric cancer for 10 years. Cox-proportional hazards model will be used to calculate hazard ratios (HRs) with $95 \%$ confidence interval. The HR of the cumulative incidence of the low-risk group on the 3 rd assessment round (P2) will be calculated refereed to total screening group on the 2 nd assessment round (P1) (Figure 1). The HR of the cumulative gastric cancer incidence in the low-risk group will be also calculated referred to the high-risk screening group at 3,5 and 10 years. 
Table 1 Sample size calculation based on 2 scenarios

\begin{tabular}{lcc}
\hline Scenario & 1 & 2 \\
\hline Cut-off value* $^{*}$ & C-II & O-I \\
Ratio, high-risk group: low-risk group & $50: 50$ & $30: 70$ \\
$\begin{array}{l}\text { Cumulative incidence in the high-risk } \\
\text { group at P3 in Figure 1 (\%) }\end{array}$ & $0.50 \%$ & $0.70 \%$ \\
$\begin{array}{l}\text { Cumulative incidence in the low-risk } \\
\text { group at P2 in Figure 1 (\%) }\end{array}$ & $0.25 \%$ & $0.30 \%$ \\
$\begin{array}{l}\text { Sample size } \\
\text { * the cut-off value was defined by grading of atrophic gastritis }\end{array}$ \\
$\begin{array}{l}\text { using the classification of Kumara and Takemoto (11). C2: close } \\
\text { type 2; O1: open type 1. }\end{array}$
\end{tabular}

\section{Discussion}

Endoscopic screening has been expected to reduce mortality from gastric cancer in East Asian countries which continues to bear a heavy burden of gastric cancer (14). Distinctively, Korea and Japan have national cancer screening programs whereas some countries have opportunistic or researchbased screening programs (2,3,15-17). In Japan, gastric cancer screening has been considered to play the main role in cancer prevention $(2,3)$. Although endoscopic screening has gradually been disseminated nationwide, there is a huge disparity in endoscopic screening access between urban and regional areas, and rapid dissemination is difficult (6). To achieve the goal for mortality reduction from gastric cancer, equal access to endoscopic screening should be provided.

Recently, the HPV test has been adopted to cervical cancer screening in the U.S. and some countries (18-20). This screening interval of the HPV test can be expanded by as much as twice that of cytological screening. This screening interval expansion has been suggested by cohort studies conducted in the U.S. and the Netherlands $(20,21)$. In cervical cancer screening, the cumulative incidence of cervical cancer has been compared between women with negative human papillomavirus (HPV) test results and women with negative cytological test results. Particularly in Dutch and the U.S. studies, the cumulative incidence of cervical cancer among HPV-negative women on the 2nd screening round was similar to that among cytologynegative women on the $3 \mathrm{rd}$ screening round $(20,21)$. These results suggest that the HPV test could predict more adequately the development of cervical cancer than cytology and that HPV-negative results indicate a longer period of being free from cervical cancer than cytology-negative results. Thus, if the HPV test is adopted for cervical cancer screening, the screening interval can be expanded by as much as twice the regular screening interval. In essence, the same theory can be adapted to enable risk stratification for gastric cancer and expand the endoscopic screening interval for gastric cancer.

Gastric cancer remains a heavy public health burden in Japan. Thus, there is a continuing need for gastric cancer screening. In such screening, a high participation rate of the target population is required to reduce mortality from gastric cancer. The recent introduction of endoscopic screening for gastric cancer as a national program is aimed at helping to improve the participation rate. However, a greater increase in participation should be required to achieve this goal. Then, in order to help build capacity for endoscopic screening, one of the potential solutions could be expand the screening interval for the low-risk group. The advantage to expanding the screening interval can be seen from the two perspective. First, from an individual perspective, there is a possibility of developing complications and the potential for overdiagnosis when they participate in endoscopic screening $(22,23)$. Decreasing the screening frequency can help to protect people from being exposed to these harms. From a population perspective, equal assess has been provided to all within the target population regardless of their living areas thus assisting efficient use of limited resources. As human resources remain insufficient, disparities in the use of endoscopic screening persist. To address these issues, a possible solution is to divide the intensive target group from low-risk group for gastric cancer and increase the endoscopic screening interval.

H. pylori infection plays a pivotal role in the development of gastric cancer. The infection causes atrophic gastritis which has been recognized as the main precursor lesion of gastric cancer (23). With further improvements in an endoscopic examination, atrophic gastritis could be accurately diagnosed by the usual upper gastrointestinal endoscopic examination (12). Risk stratification has been adopted in cancer screening programs by referring to individual risks $(24,25)$. A combination of the $H$. pylori antibody test and the serum pepsinogen test has been anticipated to be introduced as a primary screening method for gastric cancer in Japan (26). However, there has been no evidence to date showing that this combination reduces mortality from gastric cancer (22). Although the serum pepsinogen test is a good predictor of gastric cancer, its 
specificity is still not sufficiently high $(27,28)$. Besides, the International Agency of Research on Cancer recommended $H$. pylori screening to eradicate $H$. pylori for gastric cancer prevention based on expert opinions (29). Although the efficacy of $H$. pylori eradication has been evaluated, longterm effects remains unclear $(30,31)$. However, gastric cancer incidence after $H$. pylori eradication has been reported and these cannot be ignored (32). Although $H$. pylori eradication is covered by national health insurance in Japan, surveillance following the treatment has not been established (33). Afterwards, person following H.P pylori eradication often return to cancer screening program individually. Then, participants in endoscopic screening include individuals with various risks of gastric cancer regardless of $H$. pylori eradication. Therefore, a new risk stratification is needed for the efficient use of endoscopic screening.

To address disparities in the use of endoscopic screening, the endoscopic screening interval may be expanded, and the target age group defined. This can be achieved by applying the basic concept of $H$. pylori screening to the current endoscopic screening program. The overall of this study is to obtain relevant data that can be used to improve the efficient use of endoscopic screening for gastric cancer by referring to individual risks in Japan.

\section{Acknowledgments}

We appreciate the following main members of the local medical associations for the research collaborations for this study: Niigata (Rintaro Narisawa, Toshiyuki Kato, Masayasu Yamaguchi, Ryuichiro Ataka, Yukihiro Abe, Kenji Kuwana, and Kazutaka Fujita), Kanazawa (Atsushi Hashiba, Kenji Ohno, Hisashi Tsuchiyama, Ichiro Takahata, and Toshio Ueno), Maebashi (Hiroaki Hagiwara and Fumitaka Moki), Tottori (Katsuo Okada), Yonago (Yoneatsu Osaki, Michiko Shyabana, Masahi Fujise, and Yoshihito Nosaka), YuriHonjyo (Takuji Gotoda and Katsunori Iijima), Morioka (Koki Kubota, Ken Eizuka, and Atsushi Kano), Sendai (Katsuaki Kato and Daisuke Shibuya), Setagaya-Tokyo (Tadashi Terada and Kenichi Fukai), Yazu (Makoto Osaki), and Fukuoka (Shinji Kitagawa and Katsuya Hirakawa). We are grateful to Dr. Edward F. Barroga (http://orcid. org/0000-0002-8920-2607), Medical Editor and Professor of Academic Writing at St. Luke's International University for reviewing and editing the manuscript. We also thank Mr. Tsuyoshi Kuriwada, Ms. Yumiko Nakagawa, Ms. Ikuko Tominaga, and Ms. Junko Asai for their assistance in this study.

Funding: This study was financially supported by a government from the Japan Agency of Medical Research and Development Tokyo, Japan (Grant number: 20ck0106527h). The funder had no role in the conceptualization of the study design, data collection and analysis, decision to publish, or preparation of the manuscript.

\section{Footnote}

Conflicts of Interest: All authors have completed the ICMJE uniform disclosure form (available at http://dx.doi. org/10.21037/atm-20-5949). The authors have no conflicts of interest to declare.

Ethical Statement: The authors are accountable for all aspects of the work in ensuring that questions related to the accuracy or integrity of any part of the work are appropriately investigated and resolved. The study was approved by the Ethics Committee of the Miyagi Cancer Association (No.1612, Date of approval $21^{\text {st }}$ March 2017).

Open Access Statement: This is an Open Access article distributed in accordance with the Creative Commons Attribution-NonCommercial-NoDerivs 4.0 International License (CC BY-NC-ND 4.0), which permits the noncommercial replication and distribution of the article with the strict proviso that no changes or edits are made and the original work is properly cited (including links to both the formal publication through the relevant DOI and the license). See: https://creativecommons.org/licenses/by-nc-nd/4.0/.

\section{References}

1. National Cancer Center [Internet]. Center for Cancer Control and Information Services. 2019. Available online: https://ganjoho.jp/public/index.html.

2. Oshima A. A critical review of cancer screening programs in Japan. Int J Technol Assess Health Care 1994;10:346-58.

3. Hamashima C. Cancer screening guidelines and policy making: 15 years of experience in cancer screening guideline development in Japan. Jpn J Clin Oncol 2018;48:278-86.

4. Ministry of Health, Labour and Welfare [Internet]. Japan: Report of Health Promotion and Community Health Survey 2017. [cited 2020 Jun 30]. Available online: https:// www.e-stat.go.jp/stat-search/files? page $=1 \&$ toukei $=004500$ 
$25 \&$ tstat $=000001030884$.

5. Ministry of Health, Labour and Welfare [Internet]. Report of Medical Institution Survey 2016. [cited 2020 Jun 30]. Available from: https://www.e-stat.go.jp/stat-search/files?p age $=1$ \& toukei $=00450021 \&$ tstat $=000001030908$.

6. Hamashima C, Goto R. Potential capacity of endoscopic screening for gastric cancer in Japan. Cancer Sci 2017;108:101-7.

7. Correa P. Human gastric carcinogenesis: a multistep and multifactorial process--First American Cancer Society Award Lecture on Cancer Epidemiology and Prevention. Cancer Res 1992;52:6735-40.

8. Terasawa T, Nishida H, Kato K, et al. Prediction of gastric cancer development by serum pepsinogen test and Helicobacter pylori seropositivity in Eastern Asians: a systematic review and meta-analysis. PLoS One [Internet] 2014 oct [cited 2020 Jun 30];9:e109783. Available online: https://www.ncbi.nlm.nih.gov/pmc/articles/PMC4196955/ pdf/pone.0109783.pdf

9. Hamashima C, Fukao A. Quality assurance manual of endoscopic screening for gastric cancer in Japanese communities. Jpn J Clin Oncol 2016;46:1053-61.

10. Szende A, Oppe M, Devlin N, editors. EQ-5D value sets: Inventory, comparative review and user guide. Dordrecht: Springer; 2007.

11. Kimura K, Takemoto T. An endoscopic recognition for the atrophic border and its significance in chronic gastritis. Endoscopy 1969;1:87-97.

12. Kaji K, Hashiba A, Uotani C, et al. Grading of atrophic gastritis is useful for risk stratification in endoscopic screening for gastric cancer. Am J Gastroenterol 2019;114:71-9.

13. Kato K, Hashiba A, Uotani C, et al. Assessment of H. pylori infection rate and atrophic gastritis in individuals aged 61 years in Niigata city. J Gastrointest Cancer Screen 2018;56:432.

14. Ferlay J, Soerjomataram I, Ervik M, et al, editors. International Agency for Research on Cancer.

GLOBOCAN 2012: Estimated cancer incidence, mortality and prevalence worldwide in 2012 v1.0. IARC Cancer Base No. 11, Lyon. 2013. Available online: http://gco.iarc.fr/.

15. Kim Y, Jun JK, Choi KS, et al. Overview of the national cancer screening programme and the cancer screening status in Korea. Asian Pac J Cancer Prev 2011;12:725-30.

16. Lee YC, Chen TH, Chiu HM, et al. The benefit of mass eradication of Helicobacter pylori infection: a community-based study of gastric cancer prevention. Gut 2013;62:676-82.
17. Chen Q, Yu L, Hao CQ, Wang JW, et al. Effectiveness of endoscopic gastric cancer screening in a rural area of Linzhou, China: results from a case-control study. Cancer Med 2016;5:2615-22.

18. US Preventive Services Task Force, Curry SJ, Krist AH, Owens DK, et al. Screening for cervical cancer: US preventive services task force recommendation statement. JAMA 2018;320:674-86.

19. Cancer Council Australia (2016) [Internet]. National Cervical Screening Program: Guidelines for the management of screen-detected abnormalities, screendetected abnormalities, screening in specific populations and investigation of abnormal vaginal bleeding. [cited 2020 Jun 30] Available online: http://www.cancerscreening.gov. au/internet/screening/publishing.nsf/Content/healthcareproviders.

20. Huijsmans CJ, Geurts-Giele WR, Leeijen C, et al. HPV prevalence in the Dutch cervical cancer screening population (DuSC study): HPV testing using automated HC2, Cobas and Aptima workflows. BMC Cancer 2016;16:922.

21. Katki HA, Kinney WK, Fetterman B, et al. Cervical cancer risk for women undergoing concurrent testing for human papillomavirus and cervical cytology: a populationbased study in routine clinical practice. Lancet Oncol 2011;12:663-72.

22. Hamashima C. Update version of the Japanese guidelines for gastric cancer screening. Jpn J Clin Oncol 2018;48:673-83.

23. Hamashima C. Overdiagnosis of gastric cancer by endoscopic screening. World J Gastrointest Endosc 2017;9:55-60.

24. Chiu SY, Chuang SL, Chen SL, et al. Faecal haemoglobin concentration influences risk prediction of interval cancers resulting from inadequate colonoscopy quality: analysis of the Taiwanese Nationwide Colorectal Cancer Screening Program. Gut 2017;66:293-300.

25. Yousaf-Khan U, van der Aalst C, de Jong PA, et al. Risk stratification based on screening history: the NELSON lung cancer screening study. Thorax 2017;72:819-24.

26. Miki K. Gastric cancer screening by combined assay for serum anti-Helicobacter pylori IgG antibody and serum pepsinogen levels “ABC method”. Proc Jpn Acad Ser B Phys Biol Sci 2011;87:405-14.

27. Yoshida T, Kato J, Inoue I, et al. Cancer development based on chronic active gastritis and resulting gastric atrophy as assessed by serum levels of pepsinogen and Helicobacter pylori antibody titer. Int J Cancer 
2014; 134:1445-57.

28. Hamashima C, Sasazuki S, Inoue M, et al. Receiver operating characteristic analysis of prediction for gastric cancer development using serum pepsinogen and Helicobacter pylori antibody tests. BMC Cancer 2017;17:183.

29. IARC Helicobacter pylori Working Group. Helicobacter pylori eradication as a strategy for preventing gastric cancer: IARC Working Group Report Volume 8. International Agency for Research on Cancer. Lyon: 2014.

30. Ford AC, Forman D, Hunt RH, et al. Helicobacter pylori eradication therapy to prevent gastric cancer in healthy asymptomatic infected individuals: systematic review

Cite this article as: Hamashima C, Yoshimura K, Fukao A. A study protocol for expanding the screening interval of endoscopic screening for gastric cancer based on individual risks: prospective cohort study of gastric cancer screening. Ann Transl Med 2020;8(23):1604. doi: 10.21037/atm-20-5949 and meta-analysis of randomized controlled trials. BMJ 2014;348:g3174.

31. Terasawa T, Hamashima C, Kato K, et al. Helicobacter pylori eradication treatment for gastric carcinoma prevention in asymptomatic or dyspeptic adults: systematic review and Bayesian meta-analysis of randomised controlled trials. BMJ Open 2019;9:e026002.

32. Shichijo S, Hirata Y. Characteristics and predictors of gastric cancer after Helicobacter pylori eradication. World J Gastroenterol 2018;24:2163-72.

33. Sakitani K, Nishizawa T, Arita M, et al. Early detection of gastric cancer after Helicobacter pylori eradication due to endoscopic surveillance. Helicobacter 2018;23:e12503. 